\title{
Cardiac Sarcoidosis: Is it More Common in Men?
}

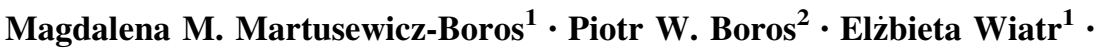 \\ Anna Kempisty ${ }^{3} \cdot$ Dorota Piotrowska-Kownacka $^{4} \cdot$ Kazimierz Roszkowski-Śliż $^{1}$
}

Received: 26 June 2015/ Accepted: 18 September 2015/Published online: 28 September 2015

(C) The Author(s) 2015

\begin{abstract}
Introduction Sarcoidosis is a systemic granulomatous disease which predominantly affects the lungs, although granulomas can also involve all other organs, including the heart. Cardiac sarcoidosis (CS) may occur at any stage of the disease and may be the cause of sudden cardiac death, even in a previously asymptomatic patient. The aim of this study was to evaluate the incidence of CS in a large group of patients diagnosed or followed up due to sarcoidosis.

Methods We performed a retrospective analysis of patients at our institution discharged with the final diagnosis "sarcoidosis" (ICD-10: D86) from January 2008 to October 2012. Only those with biopsy (from respiratory tract or lymph nodes) confirmed diagnosis of sarcoidosis were included. We then selected the subset of patients with cardiac involvement due to sarcoidosis confirmed by positive magnetic resonance imaging.

Results The study covered 1375 consecutive sarcoidosis patients ( $51 \%$ men), who were hospitalized during 5 years. Multiorgan disease was detected in 160 cases (11.7\%), and cardiac involvement was found in 64 patients $(4.7 \%$ of all), $70.3 \%$ of whom were men. Twelve of those with CS
\end{abstract}

Magdalena M. Martusewicz-Boros

m.martusewicz@gmail.com

1 3rd Lung Diseases Department, National Research Institute of TB \& Lung Diseases, Plocka 26, 01-138 Warsaw, Poland

2 Lung Pathophysiology Department, National Research Institute of TB \& Lung Diseases, Plocka 26, 01-138 Warsaw, Poland

3 1st Lung Diseases Department, National Research Institute of TB \& Lung Diseases, Plocka 26, 01-138 Warsaw, Poland

4 1st Department of Clinical Radiology, Medical University of Warsaw, Chalubinskiego 5, 02-004 Warsaw, Poland were in stage I, 48 in stage II, and four in stage III. The odds ratio for having cardiac involvement in men compared to women was 2.3 (95\% CI 1.36-4.0, $p=0.002$ ).

Conclusions Cardiac involvement in sarcoidosis was diagnosed in the similar percentage as in previously published data but was significantly more frequently in men.

Keywords Sarcoidosis - Heart involvement in systemic diseases · Cardiac sarcoidosis - Sex distribution . Epidemiology

\section{Introduction}

Sarcoidosis is a systemic granulomatous disease with predominant manifestation in the chest, often presenting as bilateral hilar lymphadenopathy with or without pulmonary infiltrates. Despite numerous hypotheses, the etiology of sarcoidosis still remains unclear. An unspecified antigen in individuals genetically predisposed induces an abnormal immune response. The consequence is an inflammatory process with formation of sarcoidosis granulomas. While more than $90 \%$ of patients with sarcoidosis have lung involvement, granulomas can also involve any other organs, including the heart [1].

Cardiac sarcoidosis (CS) is usually ascertained in about $5 \%$ of patients with already diagnosed sarcoidosis of the respiratory system but may be detected without pulmonary disease $[2,3]$. An autopsy study reported cardiac involvement in $27 \%$ of cases in United States or Europe [3-6]. These differences demonstrate the diagnostic difficulties, which are still present. Heart involvement is the primary cause of poor outcome in sarcoidosis, as may be the cause of sudden cardiac death, even in previously asymptomatic patients. CS may also cause heart failure. Early diagnosis 
of the CS and the early initiation of corticosteroid therapy seem to improve the prognosis [7-9]; however, extent involvement may correlate with the absence of functional improvement and high incidence of adverse outcomes [10]. Age gender and race have all been identified as factors associated with specific clinical presentations of sarcoidosis; however, demographic features that predict cardiac involvement remain elusive [3].

The National Tuberculosis \& Lung Diseases Research Institute in Warsaw, Poland serves as the regional referral center for patients with sarcoidosis and other interstitial lung diseases. This provided the opportunity to describe the incidence of clinically important cardiac involvement in a much larger group of patients with sarcoidosis than previously described [11-15].

The main purpose of this investigation was to evaluate the incidence of CS in a large group of patients diagnosed or followed up due to sarcoidosis and looking for specific pattern of patients diagnosed with heart involvement.

\section{Materials and Methods}

We performed retrospective analysis of database discharged patients with the final diagnosis "Sarcoidosis of other and combined sites" (ICD-10: D86.8). Only patients with biopsy (from lungs or lymph nodes) confirmed diagnosis of sarcoidosis were included. We than selected the subset of patients with cardiac involvement due to sarcoidosis, who were identified when partially or fully complied with criteria presented in Modified Guidelines for Diagnosis of Cardiac Sarcoidosis based on the Study Report on Diffuse Pulmonary Diseases from the Japan Ministry of Health and Welfare, 1993 (9) and modifications JMHW 2006 (Table 1). However, the diagnosis was confirmed finally by a positive magnetic resonance imaging (MRI) test.

The analysis covered the period from January 2008 to October 2012 in 1st and 3rd Lung Diseases Departments of the National Tuberculosis \& Lung Diseases Research Institute in Warsaw. We collected demographic information, medical history, including comorbidities, and medical treatment. Patients with a history of coronary artery disease (CAD) or other heart disease which could influence the MRI examination making it non-conclusive were not included. However, in a few cases, when MRI investigation was suggestive for CAD, but without previous history, coronary angiography was performed (in all cases it was negative and patients were included into study). The evaluation of all patients included laboratory tests (e.g., among others determination of the highest serum levels of angiotensin-converting enzyme, calcium metabolism), radiological examination, electrocardiography (ECG) and often echocardiography (ECHO), pulmonary function tests, and an ophthalmology assessment (or other consultation of specialist, if needed).

Cardiac evaluations were usually initiated because of tachycardia, confusion, syncope, chest discomfort, or

Table 1 Revised guidelines for diagnosing CS 2006 (Japan Society of Sarcoidosis and Other Granulomatous Disorders)

\footnotetext{
1. Histologic diagnosis group

Cardiac sarcoidosis is confirmed when myocardial biopsy specimens demonstrate noncaseating epithelioid cell granulomas with histological or clinical diagnosis of extracardiac sarcoidosis.

2. Clinical diagnosis group

Cardiac sarcoidosis is diagnosed in the absence of a cardiac biopsy when extracardiac sarcoidosis is diagnosed histologically or clinically and satisfies the following conditions and more than one in six basic diagnostic criteria.

(1) More than two of four major criteria are satisfied, or

(2) One in four major criteria and more than two in five minor criteria are satisfied.

Major criteria

(a) Advanced AV block

(b) Basal thinning of the interventricular septum

(c) Positive cardiac gallium uptake

(d) Left ventricle ejection fraction less than $50 \%$

Minor criteria

(a) Abnormal ECG findings: Ventricular arrhythmias (VT, multifocal or frequent PVCs), CRBBB, axis deviation or abnormal Q-wave

(b) Abnormal echocardiography: Regional abnormal wall motion or morphological abnormality (ventricular aneurysm, wall thickening)

(c) Perfusion defect detected by $201 \mathrm{Tl}$ myocardial scintigraphy or 99Tc myocardial scintigraphy

(d) Gd-enhanced MRI: Delayed enhancement of myocardium.

(e) Endomyocardial biopsy: Interstitial fibrosis or monocyte infiltration over moderate grade
}

$A V$ atrioventricular, ECG electrocardiogram, $V T$ ventricular tachycardia, $P V C s$ premature ventricular contractions, $M R I$ magnetic resonance imaging, $C R B B B$ complete right bundle branch block 
fatigue; or abnormalities seen on an ECG or ECHO. In cases with suspected CS, 24 h-Holter monitoring and delayed enhancement cardiovascular MRI were performed using a 1.5 T scanner (GE Signa Excite 11) using a torso phased-array coil. Each MRI included cine imaging with steady-state free precession sequence (SSFP; FIESTA), black blood imaging with FSE-XL breath-hold sequences (double and triple inversion recovery (IR) pre-pulse), and delayed enhancement imaging using T1-weighted, IR gradient echo sequence 15-20 min after intravenous administration of $0.1 \mathrm{mmol} / \mathrm{kg}$ Gd-based contrast agent. Images were obtained in standard long axis, short axis, and four-chamber views using ECG gating. All tests were performed using the same equipment/procedure, and results were described by the same well experienced specialist.

The nature of this study is a non-interventional, descriptive, retrospective analysis of de-identified data, obviating the need for approval from local Ethics Committee.

\section{Statistical Analysis}

Descriptive data were presented as mean $\pm \mathrm{SD}$ and range or $90 \% \mathrm{CI}$ where indicated. Group comparisons were made using paired t-tests for independent samples. The incidence ratios were presented as numbers of patients in groups and percentages. The $\chi^{2}$ test was used to test for differences in the prevalence of observations. Statistical analyses were performed using STATISTICA (data analysis software system StatSoft, Inc. 2010), version 9.1.

\section{Results}

During the 5-year period, 1375 consecutive sarcoidosis patients were observed in two lung disease departments. The distribution of gender in the whole group was comparable, there were 671 female $(49 \%)$ and 704 males $(51 \%)$, in mean age: $43.7 \pm 12.2$ years $(\mathrm{F}: 48.0 \pm 12.8$; M: $39.6 \pm 10.1)$. Multiorgan disease was detected in 160 cases $(11.7 \%)$. Cardiac involvement confirmed by MRI was found in 64 patients (4.7\% of the entire group). See Fig. 1 part A for flowchart of the study and part B for distribution of patients.

The most frequent sites, other than lung or heart involvement, were liver $(18.8 \%)$, spleen $(18.8 \%)$, and peripheral lymph nodes $(17.2 \%)$. The median time from the first diagnosis of sarcoidosis to detection of heart involvement was 18.8 months (range 0-9 years). All of the CS patients had affected lungs and/or intrathoracic lymph nodes. Twelve patients were in stage I, 48 in stage II, and four in stage III.
In $19(29.7 \%)$ cases, CS was diagnosed with radiological progression in the lungs, in 11 cases $(17.2 \%)$ during regression and in $34(53.1 \%)$ at stabilization. 16 (25\%) patients had been previously treated with corticosteroids. $50 \%$ of CS group (21 males and 11 females) were diagnosed because of symptoms (Table 2), while the others were identified due to ECG or ECHO abnormalities.

The mean age of those with CS was 47 years (range: 25-77). There were 45 males and 19 females (70.3/29.7\%) in CS group. Odds ratio for having cardiac involvement in males comparing to females was 2.3 ; $95 \%$ CI 1.4-4.0, $p=0.002$ (see Fig. 2).

\section{Discussion}

The diagnosis of CS remains difficult. There are no specific symptoms or signs. Most often heart involvement is clinically "silent" in the early stage of the disease. The initial presentation can be malignant arrhythmias, progressive heart failure, or sudden death [16-19]. This location of changes significantly worsens the prognosis of patients with sarcoidosis [20]. CS accounts for as many as $13-25 \%$ (in United States) to 58-85\% of deaths (reported in Japan) from sarcoidosis $[6,21]$. In addition to the asymptomatic course of the disease, also widely available tests like ECG or ECHO could be normal in the early stage of CS. Some cases with advanced heart involvement diagnosed postmortem had normal ECGs before death [6]. ECG changes, if any present, are nonspecific. Conduction aberrations (from incomplete and complete bundle branch block 12-61\% to atrioventricular block of any degree 26-62\%) and (ventricular) arrhythmias (2-42\%) seem to be common [8, 11, 14, 22]. Holter monitoring is useful in clinical suspicion of CS [12]. Changes in ECHO can be seen with disease progression in 14-56 \% [22-25]. Endomyocardial biopsy, despite high specificity, slack sensitivity (about 36\%), probably because of patchy involvement [26]. That is why this invasive procedure should not be a part of routine evaluation for patients with suspected CS [27].

No recommendations for the diagnosis and treatment of CS have been widely accepted. The diagnostic criteria developed by the Japanese Ministry of Health and Welfare (JMHW) in the absence of endomyocardial biopsy propose to identify cardiac involvement (Table 1). The development of imaging techniques allows the identification of changes at an earlier, less advanced stage of the disease [7, 13, 22, 28, 29]. Delayed enhancement cardiac MRI is regarded as very helpful and promising, and begins to pretend to be the gold standard for the diagnosis CS [7, 30, 31]. Recently published data and expert opinion also recommend use of MRI and positron emission tomography- 
Fig. 1 a The flowchart of the study. b The distribution of diagnosis of sarcoidosis according to ICD-10 classification, where D86.0 Sarcoidosis of lung; D86.1Sarcoidosis of lymph nodes; D86.2-Sarcoidosis of lung with sarcoidosis of lymph nodes; D86.8-Sarcoidosis of other and combined sites; D86.9-Sarcoidosis unspecified; $\mathrm{CS}(+)$-patients with diagnosis of cardiac sarcoidosis; CS(-) - patients without diagnosis of $\mathrm{CS}$, data presented as numbers of cases and percentages
(A)

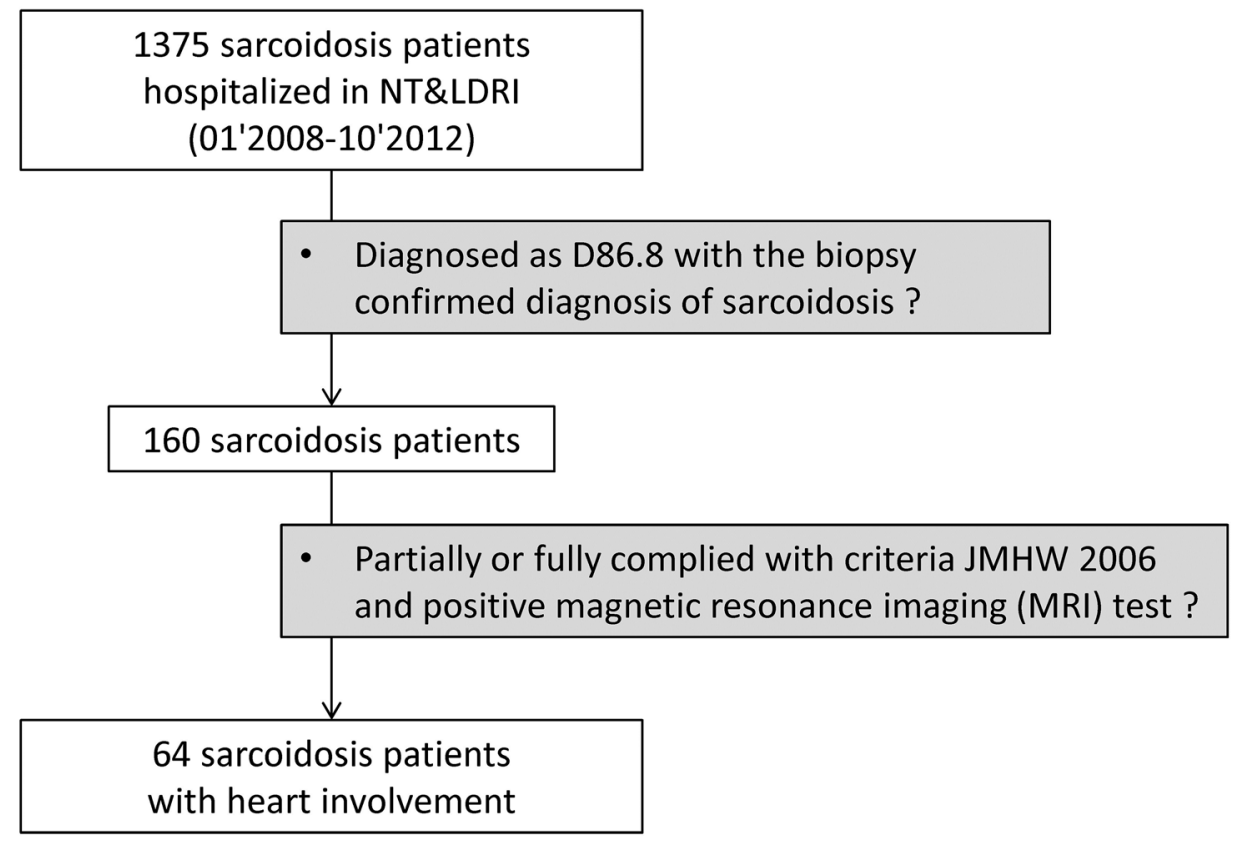

(B)

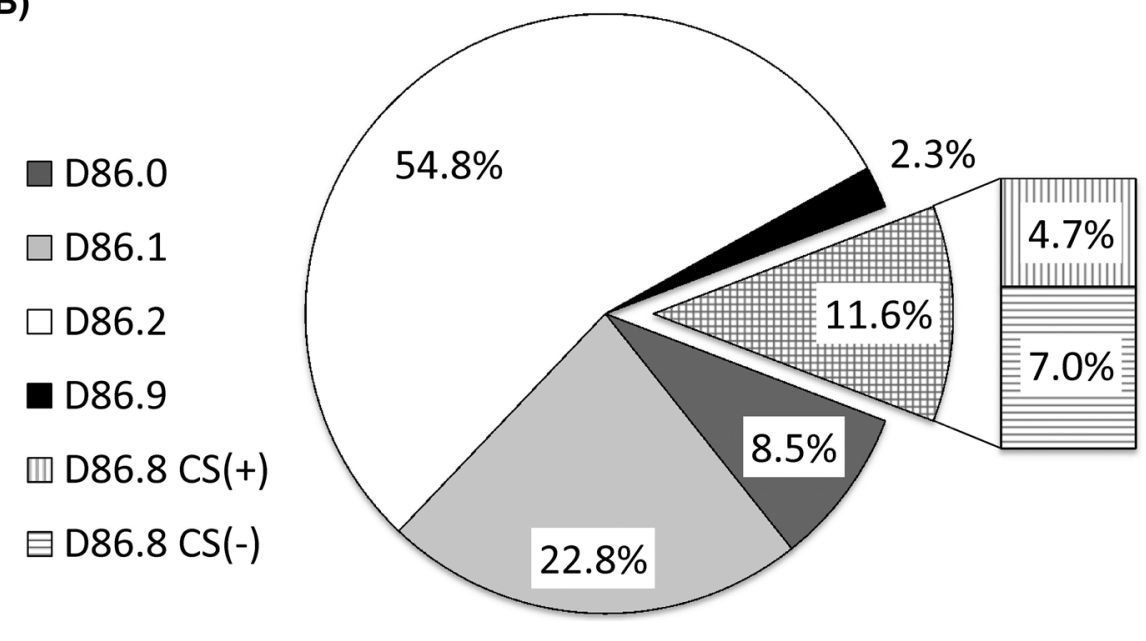

Table 2 Symptoms reported by patients with recognized CS

\begin{tabular}{lll}
\hline Symptoms & $\begin{array}{l}\text { Number of symptomatic } \\
\text { CS males }=21 / 45(46.6 \%)\end{array}$ & $\begin{array}{l}\text { Number of symptomatic } \\
\text { CS females = 11/19 (57.8 \%) }\end{array}$ \\
\hline Sudden cardiac arrest & $2(4.4 \%)$ & 0 \\
Syncope & $3(6.6 \%)$ & $3(15.8 \%)$ \\
Palpitations & $9(20 \%)$ & $7(36.8 \%)$ \\
Non-specific symptoms & $7(15.5 \%)$ & $4(21 \%)$ \\
Dyspnoea & $4(8.8 \%)$ & $2(10.5 \%)$ \\
Non-specific chest complaints & $4(8.8 \%)$ & 0 \\
\hline
\end{tabular}

computed tomography (PET-CT) as useful tools for detecting CS [32-34].

The incidence of CS in our study was lower than previously reported in postmortem or contrast-enhanced MRI based studies [5, 30, 35-38]. However, it should be noted that our study did not screen asymptomatic patients for CS but analyzed data only from symptomatic patients. Our study is the first epidemiological evaluation suggesting that CS is 
Fig. 2 Sex distribution in all patients and in CS group (numbers and percentages) all sarcoidosis patients

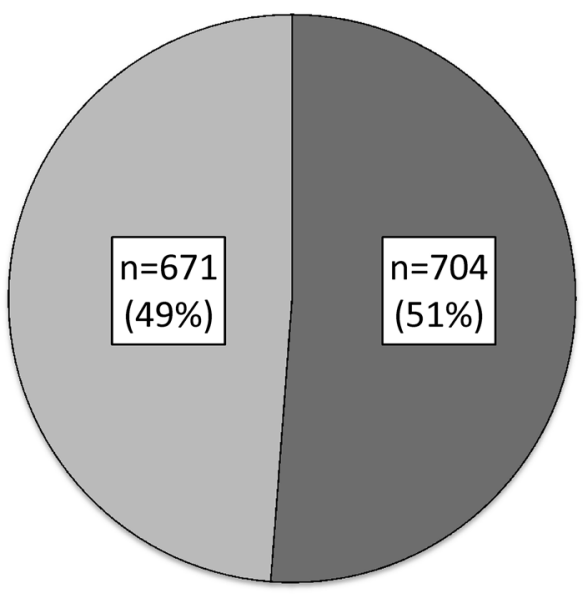

sarcoidosis patients with heart involvement

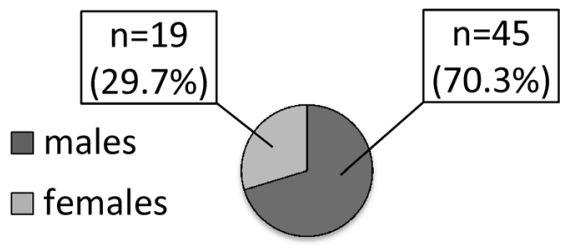

Odds ratio 2.3433

$95 \% \mathrm{Cl} 1.3559$ to 4.0496

z statistic 3.051

Significance level $P=0.0023$ more common in men than women. A Japanese study reported a predominance of CS in women [39]. Other studies have shown no difference in CS rates between men and women [14, 40, 41], so our results may not be generalizable to patients from other countries. However, in recently published study performed in Europe, results were quite similar (male/female ratio $74 / 26 \%$ ), but due to smaller size of the group and male/female disproportion, this finding could not be regarded as statistically significant [36].

\section{Limitations of the Study}

This is a retrospective review, with its inherent limitations. Sarcoidosis patients included to the study were hospitalized (no out-patients) in a large tertiary specialist center, what rise concern about pre-selection and possible bias; however, in Polish realities of health care funding, almost all patients are diagnosed in hospitals due to poor financing of diagnostic procedures in out-patients clinics. We did not perform coronary angiography studies to exclude CAD as a cause for delayed myocardial enhancement in all patients; however, patients with known history of CAD were excluded from the study. As only one person evaluated the MRI study inter-observer, and intra-observer agreement was not assessed.

\section{Conclusions}

Clinically evident cardiac involvement in sarcoidosis was diagnosed in the similar percentage as in previously published data but was significantly more frequently in men. Further investigations are needed for factors predisposing to the occurrence of cardiac involvement in sarcoidosis and gender should be considered.

Acknowledgments Acknowledgements for all colleagues who participated in the diagnosis and care of patients.

Authors Contributions MM Martusewicz-Boros conception and design, acquisition of data, analysis and interpretation of data, draft of the manuscript, revision it critically for important intellectual content; final approval of the version to be published. PW Boros, D Piotrowska-Kownacka analysis and interpretation of data, revision manuscript critically for important intellectual content; final approval of the version to be published. E Wiatr, A Kempisty, K RoszkowskiŚliż acquisition of data, revision manuscript critically for important intellectual content; final approval of the version to be published.

\section{Compliance with Ethical Standards}

\section{Conflict of interest None.}

Open Access This article is distributed under the terms of the Creative Commons Attribution 4.0 International License (http://crea tivecommons.org/licenses/by/4.0/), which permits unrestricted use, distribution, and reproduction in any medium, provided you give appropriate credit to the original author(s) and the source, provide a link to the Creative Commons license, and indicate if changes were made.

\section{References}

1. Statement on sarcoidosis (1999) Joint Statement of the American Thoracic Society (ATS), the European Respiratory Society (ERS) and the World Association of Sarcoidosis and Other Granulomatous Disorders (WASOG) adopted by the ATS Board of Directors and by the ERS Executive Committee, February 1999. Am J Respir Crit Care Med 160:736-755

2. Nelson JE, Kirschner PA, Teirstein AS (1996) Sarcoidosis presenting as heart disease. Sarcoidosis Vasc Diffuse Lung Dis 13:178-182 
3. Baughman RP, Teirstein AS, Judson MA et al (2001) Clinical characteristics of patients in a case control study of sarcoidosis. Am J Respir Crit Care Med 164:1885-1889

4. Sharma OP, Maheshwari A, Thaker K (1993) Myocardial sarcoidosis. Chest J 103:253-258

5. Roberts WC, McAllister HA Jr, Ferrans VJ (1977) Sarcoidosis of the heart. A clinicopathologic study of 35 necropsy patients (group 1) and review of 78 previously described necropsy patients (group 11). Am J Med 63:86-108

6. Silverman KJ, Hutchins GM, Bulkley BH (1978) Cardiac sarcoid: a clinicopathologic study of 84 unselected patients with systemic sarcoidosis. Circulation 58:1204-1211

7. Mantini N, Williams B Jr, Stewart J et al (2012) Cardiac sarcoid: a clinician's review on how to approach the patient with cardiac sarcoid. Clin Cardiol 35:410-415

8. Yazaki Y, Isobe M, Hiroe M et al (2001) Prognostic determinants of long-term survival in Japanese patients with cardiac sarcoidosis treated with prednisone. Am J Cardiol 88:1006-1010

9. Bargout R, Kelly RF (2004) Sarcoid heart disease: clinical course and treatment. Int J Cardiol 97:173-182

10. Ise $T$, Hasegawa $T$, Morita $Y$ et al (2014) Extensive late gadolinium enhancement on cardiovascular magnetic resonance predicts adverse outcomes and lack of improvement in LV function after steroid therapy in cardiac sarcoidosis. Heart 100:1165-1172

11. Chapelon-Abric C, de Zuttere D, Duhaut P et al (2004) Cardiac sarcoidosis: a retrospective study of 41 cases. Medicine (Baltimore) 83:315-334

12. Mehta D, Lubitz SA, Frankel Z et al (2008) Cardiac involvement in patients with sarcoidosis: diagnostic and prognostic value of outpatient testing. Chest 133:1426-1435

13. Patel MR, Cawley PJ, Heitner JF et al (2009) Detection of myocardial damage in patients with sarcoidosis. Circulation 120:1969-1977

14. Smedema JP, Snoep G, van Kroonenburgh MP et al (2005) Cardiac involvement in patients with pulmonary sarcoidosis assessed at two university medical centers in the Netherlands. Chest 128:30-35

15. Judson MA, Baughman RP, Teirstein AS et al (1999) Defining organ involvement in sarcoidosis: the ACCESS proposed instrument. ACCESS Research Group. A Case Control Etiologic Study of Sarcoidosis. Sarcoidosis Vasc Diffuse Lung Dis $16: 75-86$

16. Youssef G, Beanlands RS, Birnie DH et al (2011) Cardiac sarcoidosis: applications of imaging in diagnosis and directing treatment. Heart 97:2078-2087

17. Doughan AR, Williams BR (2006) Cardiac sarcoidosis. Heart 92:282-288

18. Martusewicz-Boros M, Wiatr E, Piotrowska-Kownacka D et al (2007) Cardiac sarcoidosis—own experiences. Pneumonol Alergol Pol 75:100-107

19. Martusewicz-Boros M (2006) Cardiac sarcoidosis. Pol Arch Med Wewn 116:1207-1212

20. Yeboah J, Lee C, Sharma OP (2011) Cardiac sarcoidosis: a review 2011. Curr Opin Pulm Med 17:308-315

21. Iwai K, Sekiguti M, Hosoda Y et al (1994) Racial difference in cardiac sarcoidosis incidence observed at autopsy. Sarcoidosis $11: 26-31$

22. Nunes H, Freynet O, Naggara $\mathrm{N}$ et al (2010) Cardiac sarcoidosis. Semin Respir Crit Care Med 31:428-441
23. Fahy GJ, Marwick T, McCreery CJ et al (1996) Doppler echocardiographic detection of left ventricular diastolic dysfunction in patients with pulmonary sarcoidosis. Chest 109:62-66

24. Lewin RF, Mor R, Spitzer S et al (1985) Echocardiographic evaluation of patients with systemic sarcoidosis. Am Heart J 110:116-122

25. Burstow DJ, Tajik AJ, Bailey KR et al (1989) Two-dimensional echocardiographic findings in systemic sarcoidosis. Am J Cardiol 63:478-482

26. Uemura A, Morimoto S, Hiramitsu S et al (1999) Histologic diagnostic rate of cardiac sarcoidosis: evaluation of endomyocardial biopsies. Am Heart J 138:299-302

27. Kim JS, Judson MA, Donnino R et al (2009) Cardiac sarcoidosis. Am Heart J 157:9-21

28. Osman F, Foundon A, Leyva P et al (2008) Early diagnosis of cardiac sarcoidosis using magnetic resonance imaging. Int $\mathrm{J}$ Cardiol 125:e4-e5

29. Manins V, Habersberger J, Pfluger H et al (2009) Cardiac magnetic resonance imaging in the evaluation of cardiac sarcoidosis: an Australian single-centre experience. Intern Med J 39:77-82

30. Smedema JP, Snoep G, van Kroonenburgh MP et al (2005) Evaluation of the accuracy of gadolinium-enhanced cardiovascular magnetic resonance in the diagnosis of cardiac sarcoidosis. J Am Coll Cardiol 45:1683-1690

31. Tadamura E, Yamamuro M, Kubo S et al (2005) Effectiveness of delayed enhanced MRI for identification of cardiac sarcoidosis: comparison with radionuclide imaging. AJR Am J Roentgenol 185:110-115

32. Birnie DH, Sauer WH, Bogun F et al (2014) HRS expert consensus statement on the diagnosis and management of arrhythmias associated with cardiac sarcoidosis. Heart Rhythm 11:1305-1323

33. Miyagawa M, Yokoyama R, Nishiyama Y et al (2014) Positron emission tomography-computed tomography for imaging of inflammatory cardiovascular diseases. Circ J 78:1302-1310

34. Judson MA, Costabel U, Drent M et al (2014) The WASOG Sarcoidosis Organ Assessment Instrument: an update of a previous clinical tool. Sarcoidosis Vasc Diffuse Lung Dis 31:19-27

35. Nagai T, Kohsaka S, Okuda $S$ et al (2014) Incidence and prognostic significance of myocardial late gadolinium-enhancement in sarcoidosis patients without cardiac manifestation. Chest 146(4):1064-1072

36. Greulich S, Deluigi CC, Gloekler S et al (2013) CMR imaging predicts death and other adverse events in suspected cardiac sarcoidosis. JACC Cardiovasc Imaging 6:501-511

37. Patel AR, Klein MR, Chandra S et al (2011) Myocardial damage in patients with sarcoidosis and preserved left ventricular systolic function: an observational study. Eur J Heart Fail 13:1231-1237

38. Cheong BY, Muthupillai R, Nemeth M et al (2009) The utility of delayed-enhancement magnetic resonance imaging for identifying nonischemic myocardial fibrosis in asymptomatic patients with biopsy-proven systemic sarcoidosis. Sarcoidosis Vasc Diffuse Lung Dis 26:39-46

39. Yoshida Y, Morimoto S, Hiramitsu S et al (1997) Incidence of cardiac sarcoidosis in Japanese patients with high-degree atrioventricular block. Am Heart J 134:382-386

40. Morimoto T, Azuma A, Abe S et al (2008) Epidemiology of sarcoidosis in Japan. Eur Respir J 31:372-379

41. Rybicki BA, Major M, Popovich J Jr et al (1997) Racial differences in sarcoidosis incidence: a 5-years study in a health maintenance organization. Am J Epidemiol 145:234-241 